\title{
Article
}

\section{Sleep Disorders and Risk of Incident Depression: A Population Case-Control Study}

\author{
Enda M. Byrne ${ }^{1}$, Allan Timmerman ${ }^{2,3}$, Naomi R. Wray ${ }^{1,4}$ and Esben Agerbo ${ }^{2,3,5}$ \\ ${ }^{1}$ The University of Queensland, Institute for Molecular Biosciences, Brisbane, Australia, ${ }^{2}$ Centre for Integrated Register-based Research at Aarhus University, \\ Aarhus, Denmark, ${ }^{3}$ National Centre for Register-based Research, Aarhus University, Aarhus, Denmark, ${ }^{4}$ The University of Queensland, Queensland Brain Institute, \\ Brisbane, Australia and ${ }^{5} \mathrm{PSSCH}$, Lundbeck Foundation Initiative for Integrative Psychiatric Research, Denmark
}

\begin{abstract}
We sought to investigate the risk of incident major depressive disorder (MDD) attributable to a range of sleep disorders in the Danish population. Data were obtained by linking longitudinal Danish population-based registers. A total of 65,739 individuals who had first onset of depression between 1995 and 2013 were selected as cases. For each case, a set of 20 controls of the same sex, birth month and year and who had not had depression by the date that the case was diagnosed were selected at random form the population $(N=1,307,580$ in total). We examined whether there was an increased rate of prior sleep disorders in MDD cases compared to controls using conditional logistic regression. An increased risk of incident depression in cases was found for all sleep disorders analyzed. Highest incidence rate ratios (IRRs) were found for circadian rhythm disorders (IRR = 7.06 [2.78-17.91]) and insomnia of inorganic origin $(\operatorname{IRR}=6.76$ [4.37-10.46]). The lowest estimated IRR was for narcolepsy (IRR $=2.00$ [1.26-3.17]). Those diagnosed with a sleep disorder in the last 6 months were at highest risk of developing depression compared to those with at least 1 year since diagnosis (3.10 vs. 2.36). Our results suggest that having any sleep disorder is a risk factor for incident depression. Depression screening should be considered for patients with sleep disorders, and where possible, long-term follow-up for mental health problems is advisable.
\end{abstract}

Keywords: Depression; insomnia; parasomnia; narcolepsy; sleep apnea; circadian

(Received 11 April 2019; accepted 15 April 2019)

Difficulties with sleep and disruptions to circadian rhythms are hallmarks of psychiatric disorders. In the case of mood disorders, disruptions to sleep can form part of a clinical diagnosis based on the criteria established in the Diagnostic and Statistical Manual of Mental Disorders (5th ed., DMS-V, American Psychiatric Association, 2013). Both insomnia and hypersomnia can form part of a diagnosis of major depressive disorder (MDD), the most common mood disorder (Pedersen et al., 2014). Sleep and mood are tightly linked, with mood tending to decrease as time since last sleep increases, and interventions to improve poor sleep can improve symptoms of depression (Christensen et al., 2016).

While previously seen as a symptom of MDD, there is increasing recognition that sleeping difficulties can occur prior to onset of depression and are a risk factor for MDD. A number of seminal studies have found increased rates of depression among patients with sleep disorders, including insomnia and hypersomnia (Breslau et al., 1996; Ford \& Kamerow, 1989), narcolepsy (Ohayon, 2013), obstructive sleep apnea (OSA; Peppard et al., 2006) and restless leg syndrome (Becker \& Sharon, 2014). An important aspect of treating sleep disorders is understanding the risks of developing MDD in patients and ensuring that appropriate

Author for correspondence: Enda M. Byrne, Email: enda.byrne@uq.edu.au The work was conducted at the Center for Integrated Register-based Research at Aarhus University, Aarhus, Denmark

Cite this article: Byrne EM, Timmerman A, Wray NR, and Agerbo E. (2019) Sleep Disorders and Risk of Incident Depression: A Population Case-Control Study. Twin Research and Human Genetics 22: 140-146, https://doi.org/10.1017/thg.2019.22 treatment is given. Likewise, patients presenting with MDD may have an underlying sleep disorder that when treated will lead to remittance of depression symptoms.

For some disorders such as parasomnias and circadian rhythm disorders, studies showing increased rates of depression in cases have only been correlational or cross-sectional, since longitudinal studies are costly. For other disorders such as insomnia, existing studies that have identified increased risk of depression in patients have utilized a range of epidemiological designs with different strengths and weaknesses. In some instances, the associations were evaluated in cross-sectional studies where the order of the outcome and exposure are unknown. Other studies have focused on specific age ranges or have used clinical cohorts that have been heavily ascertained for sleep disorders and may not be representative of the general population. Moreover, previous studies of the temporal relationship between sleep disorders and MDD have been limited by short follow-up periods where onset of depression has been assessed at specified intervals, rather than on a continouous basis. Adjustment for confounding factors such as cohabitation status, employment and income has also not been possible for the most part.

The aim of this study was to further our understanding of sleep disorders as a risk factor for incident depression. Specifically, we sought to estimate the increased risk of developing MDD in those with sleep disorders in an entire population. We utilize the Danish national registers, which contain records on all general and psychiatric hospital visits for the entire Danish population.

(c) The Author(s) 2019. This is an Open Access article, distributed under the terms of the Creative Commons Attribution licence (http://creativecommons.org/licenses/by/4.0/), which permits unrestricted re-use, distribution, and reproduction in any medium, provided the original work is properly cited. 


\section{Methods}

\section{Data}

Data were obtained by linking longitudinal Danish populationbased registers using the unique personal identification number assigned to all people living in Denmark since 1969 (Pedersen et al., 2006). Socioeconomic and demographic data were obtained from the Danish Civil Registration System (Pedersen, 2011) and the Danish Integrated Database for Labour Market Research (Petersson et al., 2011). Information on sleep disorders was obtained from the Danish National Hospital Register (Lynge et al., 2011) and the Danish Psychiatric Central Research Register (Mors et al., 2011) and major depression diagnoses were taken only from the latter. The former was established in 1977 and the latter in 1969. The psychiatric register is based on diagnoses made by psychiatrists according to the Danish version of the International Classification of Disease (ICD)-8 and ICD-10 classification and contains computerized records on all inpatients from 1969 to 1994, and all in- and outpatients from 1994 to 2013 (Munk-Jorgensen \& Mortensen, 1997). Likewise, the Danish National Hospital Register contains computerized records of all inpatient visits to Danish hospitals since 1977. Health care is provided free of charge to the patients in Denmark. Diagnoses of sleep disorders and major depression were only included when listed as the primary diagnosis.

Until 1994, diagnosis was given according to ICD-8 codes and from 1995 onwards according to ICD-10 codes. ICD-8 codes do not provide any information on specific diagnoses of sleep disorders; therefore, the present study includes only information on sleep disorders from 1995 onwards. Depression diagnosis is included in the ICD-8, and so data in that period were used to ensure that cases were not previously treated for depression.

\section{Study Design}

We used a nested case-control design (Borgan et al., 1995) to account for variability in depression and sleep disorder risk across ages and across time. MDD cases were defined as described in Laursen et al.'s (2016) first diagnosis of depression in the registers between 1995 and 2013 (defined as ICD-10 codes F32 and F33 and no ICD-8 codes 296.09, 296.29, 296.99, 298.09, 300.49 prior to 1995) with first recorded onset of depression between 15 and 60 years. All cases were born in Denmark and living in Denmark when becoming a case. Cases were also required to have been living in Denmark from one year before the study period (from 1994) or from when they were 15 years old, whichever came last. Lastly, cases were required to not have been diagnosed with a sleep disorder on the same day as being diagnosed with depression.

For each case, a set of 20 controls who had not been diagnosed with MDD by the diagnosis date of the case and who were matched for gender and birth month and year was selected at random from the population. Controls were also required to have been living in Denmark from the year prior to the exposure period (1994 onwards) or from age 15 onwards, whichever came latest. Individuals who subsequently went on to be diagnosed with MDD, but who did have a recorded diagnosis of depression on the day that the case of interest was diagnosed, could be included as matched controls to previously observed cases.

Sleep disorders from the F and G chapters of the ICD-10 were included as exposure variables. Disorders from the $\mathrm{F}$ chapter are classified as inorganic sleep disorders - those without a known physical cause. Disorders in the G chapter are classified as organic disorders. Supplementary Table 1 shows the ICD-10 codes that were included and how they were grouped into broad diagnoses (e.g., insomnia, hypersomnia, parasomnias).

For disorders for which there are both inorganic and organic forms, we considered both the overall broad diagnoses as well as the inorganic group and organic group if the sample size was large enough to estimate the risk ratio within a reasonably sized confidence interval. This was also partly due to privacy issues associated with the use of the registers for research that restrict analyses to sample sizes above a set threshold. We further considered all inorganic sleep disorders (all diagnoses with ICD-10 codes F50-59) and all organic sleep disorders (all diagnoses with ICD-10 codes G47.0x-G47.6x) together as risk factors. The ICD-10 codes that were considered and the diagnostic groups into which they fall are shown in Supplementary Table 1. Not all ICD-10 codes for sleep disorders were present in the register.

Socioeconomic and demographic variables were extracted for each individual in the study from the registers. We considered labour market participation, income, education, cohabiting status and degree of urbanization of place of residence from the year prior to depression onset as covariates that were adjusted for in the analysis. Employment status was divided into those in full employment, those who were unemployed but seeking work, those on leave such as family, sick or military leave, those receiving social benefits and a final group consisting of those outside the workforce but not seeking work. Income was normalized by year, birth year and gender, and divided into quintiles. Education was divided into five domains according to the highest level of education achieved. Couples who were married, in registered partnerships, or who were living together were considered as cohabiting and compared to those who were single or children living with parents.

\section{Statistical Analysis}

The incidence rate ratio (IRR) of MDD associated with sleep disorders was estimated by conditional logistic regression, with each case considered to be a separate stratum using the PHREG function in SAS 9.4. Both unadjusted IRRs and IRRs adjusted for marital status, cohabitation status, annual income (divided into quintiles by calendar year, age and sex), education level and parental psychiatric disorders were estimated. Within groups of disorders, we evaluated and compared the IRRs for sleep disorders within the six months prior to depression, disorders with onset between six months and one year prior to depression, and those occurring more than one year prior to depression. Odds ratios from a standard logistic regression of sleep disorders on depression case/control status are presented for comparison.

\section{Results}

A total of 65,379 individuals met the criteria for being a case and $1,307,580$ matched controls were included in the analysis. Supplementary Table 2 provides an overview of the sample characteristics. The sample included $62.7 \%$ women, reflecting the higher incidence of MDD in women in the Danish population (Pedersen et al., 2014). As expected based on previous studies, the risk of depression was higher in those with lower incomes, shorter time in education, those who were unemployed, not married and living in large cities (Supplementary Table 2).

The frequency of each group of sleep disorders in cases and controls as well as a selected number of subdiagnoses where there was sufficient sample size to evaluate risk of depression are provided in Table 1. Many individual diagnoses did not have enough cases to 
Table 1. Sleep disorders evaluated as risk factors for fepression and their frequency in depression cases and controls

\begin{tabular}{|c|c|c|c|c|c|c|c|c|c|}
\hline Disorder & Subdisorder/group & ICD-10 code & Cases & Controls & $\begin{array}{l}\text { Percentage } \\
\text { in cases }\end{array}$ & $\begin{array}{l}\text { Percentage in } \\
\text { Controls }\end{array}$ & $\begin{array}{l}\text { Odds ratio } \\
\text { of MDD }\end{array}$ & $\begin{array}{l}\text { Lower } \\
95 \% \mathrm{Cl}\end{array}$ & $\begin{array}{l}\text { Upper } \\
95 \% \mathrm{Cl}\end{array}$ \\
\hline \multirow{3}{*}{ Insomnia } & All & & 62 & 206 & 0.094 & 0.016 & 6.02 & 4.53 & 8.00 \\
\hline & Paradoxical insomnia & F51.03 & 17 & 39 & 0.026 & 0.003 & 8.73 & 4.94 & 15.42 \\
\hline & Organic insomnia & G47.0z & 18 & 87 & 0.027 & 0.007 & 4.14 & 2.49 & 6.88 \\
\hline Hypersomnia & Organic hypersomnia & G47.1x & 53 & 380 & 0.081 & 0.029 & 2.79 & 2.09 & 3.72 \\
\hline $\begin{array}{l}\text { Circadian rhythm sleep } \\
\text { disorders }\end{array}$ & $\begin{array}{l}\text { Nonorganic circadian } \\
\text { rhythm disorder }\end{array}$ & F51.2x & 6 & 17 & 0.009 & 0.001 & 7.07 & 2.79 & 17.92 \\
\hline Parasomnias & All & & 31 & 222 & 0.047 & 0.017 & 2.79 & 1.92 & 4.07 \\
\hline Narcolepsy & All & G47.4x & 20 & 200 & 0.030 & 0.015 & 2.00 & 1.26 & 3.17 \\
\hline \multirow[t]{2}{*}{ SDB } & All & G47.3x & 422 & 3627 & 0.642 & 0.276 & 2.32 & 2.10 & 2.57 \\
\hline & OSA & G47.33 & 45 & 245 & 0.068 & 0.019 & 3.68 & 2.67 & 5.05 \\
\hline $\begin{array}{l}\text { All inorganic sleep } \\
\text { disorders }\end{array}$ & & F51.0-F51.9 & 73 & 306 & 0.111 & 0.023 & 4.77 & 3.70 & 6.16 \\
\hline All organic disorders & & G47.0-G47.6 & 511 & 4270 & 0.777 & 0.325 & 2.39 & 2.18 & 2.62 \\
\hline All sleep disorders & & F51.0-G47.6 & 584 & 4576 & 0.888 & 0.348 & 2.55 & 2.33 & 2.78 \\
\hline
\end{tabular}

Table 2. IRRs for depression by sleep disorder classification

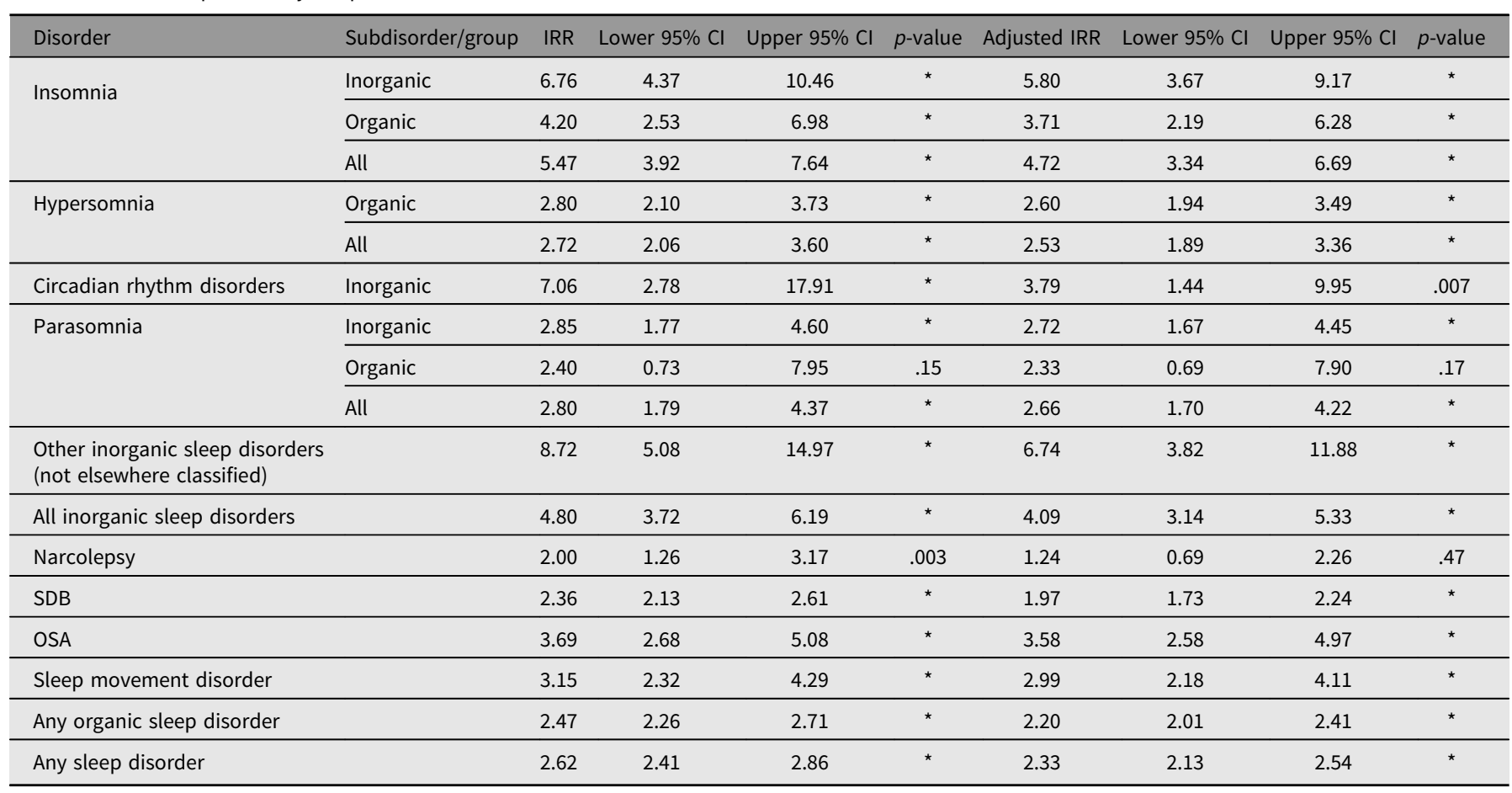

Note: ${ }^{*} p$ value $<.001$. 
Table 3. IRRs by time since diagnosis for a sleep disorder

\begin{tabular}{lccr}
\hline Disorder & $<6$ months prior & $6-12$ months prior & More than 12 months prior \\
\hline Insomnia & $11.60(5.84-23.05)$ & $3.53(1.03-12.04)$ & $3.78(2.42-5.90)$ \\
\hline Hypersomnia & $4.11(1.92-8.79)$ & $3.45(1.34-8.91)$ & $2.45(1.78-3.76)$ \\
\hline All inorganic sleep disorders & $10.69(4.98-22.95)$ & $2.61(0.78-8.69)$ & $3.26(2.36-4.51)$ \\
\hline OSA & $4.46(2.38-8.33)$ & $3.88(1.97-7.64)$ & $3.18(2.03-4.99)$ \\
\hline Any organic sleep disorder & $2.71(2.04-3.60)$ & $2.83(2.10-3.80)$ & $2.30(2.07-2.56)$ \\
\hline Any sleep disorder & $3.10(2.38-4.01)$ & $2.83(2.12-3.77)$ & $2.36(2.14-2.62)$ \\
\hline
\end{tabular}

permit estimation of IRRs for depression. In many instances, a general diagnosis code for a disorder was given in the registers. For example, the code F51.0 was the most frequently used code for insomnia, rather than the more specific codes for subtypes of insomnia such as paradoxical insomnia F51.03.

The IRRs for all of the sleep disorders examined are shown in Table 2. The IRRs were highest for inorganic circadian rhythm disorders $(\mathrm{IRR}=7.06$, adjusted $I R R=3.61)$ and inorganic insomnia ( $I R R=6.76$, adjusted IRR $=6.16)$. These results reflect a trend that inorganic disorders had a higher associated risk of developing depression than organic disorders (IRR 4.80 compared to 2.47).

The IRRs by sex for disorders with sufficient sample size to calculate them are presented in Supplementary Table 3. The most notable differences between sexes were for insomnia, where the IRR across all types of insomnia was 7.29 (4.42-12.03) for men in the unadjusted model and $4.44(2.83-6.98)$ in women, and sleep movement disorders, where the unadjusted IRR was 4.39 (2.99$6.44)$ in women and 1.97 (1.16-3.35) in men, but these differences are not statistically significant. Across all disorders, the estimated IRR for women was slightly higher in the unadjusted model -2.94 (2.55-3.38) compared to 2.47 (2.06-2.74). However, after adjusting for potential confounders, the estimated IRRs were approximately the same in both women and men - 2.37 (2.06-2.74) and 2.28 (2.04-2.55). It is important to note that the baseline risk for women is higher as MDD is more prevalent in women; therefore, equivalent IRRs will lead to a larger number of cases in women than in men.

For disorders for which there were sufficient number of cases to separate into categories according to how recently relative to the onset of depression the sleep disorder was treated, we evaluated the IRRs according to time since onset. Both inorganic and organic insomnia and hypersomnia were pooled together for this analysis. The results are presented in Table 3.

A general trend of decreasing risk for depression as the time since being diagnosed with a sleep disorder was found. For insomnia, there was a large decrease in risk after the first 6 months since diagnosis. However, even 12 months postdiagnosis, there was still at least a two-fold increased risk of incident depression.

\section{Discussion}

The relationship between sleep disorders and depression is an important area of research owing to the growing evidence that sleep problems can precede onset of psychiatric disorders. Sleep disorders are potentially critical modifiable risk factors for psychiatric disorders. If sleep problems are promptly treated, they can relieve symptoms of depression and potentially prevent development of a depressive disorder. A number of studies have attempted to estimate the increased risk to depression attributable to sleep disorders. The present study leverages population medical records to investigate the relationship and has several advantages attributable to using population medical records: inclusion of large numbers of individuals across a wide age range, avoidance of biases related to ascertainment and loss to follow-up, and no reliance on self-report information.

This is to our knowledge the first study of the longitudinal risk associated with sleep disorders in an entire population. Nearly all incident cases of depression diagnosed at a hospital in Denmark from 1995 to 2013 were included. The risk ratios between disorders can be directly compared. There is little loss to follow-up and each person can be followed for a long time after being diagnosed with a sleep disorder. There are also several limitations to this study that need to be considered and that might affect generalizability to other populations and these are discussed below.

\section{Depression and Sleep Disorders}

We found that there was an increased risk of incident depression in cases for all sleep disorders analyzed. Even without knowing the specific disorder, a patient presenting with any sleep disorder is at approximately 2.33-fold greater risk of later diagnosis of MDD. Due to limitations of the sample size and privacy concerns associated with register-based data, in most cases it was not possible to evaluate risk to depression associated with specific diagnoses, and instead disorders were grouped by broad diagnostic categories.

\section{Insomnia}

It is perhaps not surprising that one of the highest IRRs was found for insomnia, and specifically insomnia of nonorganic origin. A pathway from insomnia to depression was first established by Ford and Kamerow (1989), and since then there have been more than 40 studies evaluating the risk for depression associated with insomnia (Baglioni et al., 2011). A metaanalysis of 21 studies that evaluated risk of developing depression in insomnia patients gave an estimated odds ratio of 2.6 in a random-effects model.

In the present study, we estimate the IRR for inorganic insomnia as 6.76 (adjusted 5.80) and for insomnia of any kind 5.47 (adjusted 4.72). This estimate is higher than all of the studies included in the metaanalysis apart from the study by Perlis et al. (2006), which was conducted in elderly patients.

One possible reason why the estimated IRR is higher in this study may be due to the fact that data recorded in registers may include only very severe forms of insomnia and also severe forms of depression (general practice health records are not available). Support for this comes from the fact that the prevalences of sleep disorders are low compared to those found in other studies. 
The prevalence of insomnia in depression cases was only $0.04 \%$. This is substantially lower than the estimated prevalence of insomnia of $10-15 \%$ found in other studies, and in some instances, the prevalence of insomnia has been found to be greater than $50 \%$ in patients with depression.

\section{Hypersomnia and Narcolepsy}

The estimated IRR of hypersomnia was approximately half that of insomnia, indicating that excessive sleep is not as strong a risk factor for developing depression as insufficient sleep. The even lower IRR of narcolepsy (2.00) supports this, although narcolepsy may have different biological underpinnings than other forms of hypersomnia, making the comparison complicated.

Hypersomnia is endorsed as a symptom of depression at a much lower rate than insomnia, but daytime fatigue is common. There are few longitudinal studies of the relationship between hypersomnia and depression. Ford and Kamerow found higher rates of incident depression in those with hypersomnia compared to insomnia based on survey data (Ford \& Kamerow, 1989). Another study by Breslau et al. (1996) found a lower odds ratio of incident depression in hypersomnia compared to insomnia, a similar finding to the present study. Likewise, in a cross-sectional study of patients with sleep disorders, Vandeputte and de Weerd (2003) found that the rate of depression in hypersomnia patients was half that in insomnia patients. These differences between studies may reflect heterogeneity in assessment of hypersomnia, with some being based on self-report and others including cases diagnosed by a physician.

Cross-sectional studies of narcolepsy and depression have found increased rates of depressive symptoms in narcolepsy with cataplexy patients (Krishnan et al., 1984; Roy, 1976). But an increase in the rates of depressive disorder has not been detected (Fortuyn et al., 2010; Vourdas et al., 2002). After adjusting for confounders, the IRR in narcolepsy patients was 1.27 , not significantly different to 1 . While not ruling narcolepsy out as a risk factor for depression, these results support the hypothesis that the risk is lower than for other sleep disorders.

\section{Circadian Rhythm Disorders}

Disruptions to circadian rhythms in depression have been well documented, and patients with circadian disorders have elevated rates of depression (Germain \& Kupfer, 2008). To our knowledge, this is the first study to investigate the longitudinal relationship between circadian disruption and depression. Although limited by small sample size, our results indicate that circadian rhythm disorders are strong risk factors for developing MDD. Further investigation is required to understand how this risk is mediated. It is possible that insomnia brought about by an underlying circadian disruption could lead to increased risk of depression. More studies with larger sample sizes are needed. We were unable to investigate the relationship between specific circadian disorders and depression due to insufficient sample size.

\section{Parasomnias and Sleep Movement Disorders}

Parasomnias, including night terrors and nightmare disorder, remain understudied compared to other sleep disorders. Depressive symptoms are common in people with parasomnias (Beauchemin \& Hays, 1996; Kales et al., 1980; Llorente et al., 1992), but there have been no studies evaluating the risk of incident depression in those who suffer from them. One possible reason for this is that patients rarely present with a parasomnia without depression. We estimate the adjusted IRR of MDD in those with a parasomnia to be 2.66. This is lower than the IRR for insomnia, suggesting that periodic disruptions to sleep are not as harmful as persistent lack of sleep.

Sleep movement disorders are a broad category of disorder covering periodic leg movement disorder, leg cramps and bruxism. As yet, few studies have evaluated risk of developing depression in sleep-related movement disorders. Our findings suggest those with a movement disorder are at three-fold greater risk of developing depression and screening for depression may be warranted. Interestingly, the relative risk for women was twice as large as for men. Further studies are needed to understand why women are at increased risk.

\section{OSA and Sleep-Disordered Breathing (SDB)}

OSA is one of the most common sleep disorders and is the most common single sleep disorder found in the Danish registers. Prevalence studies have found a wide range of rates of self-report depression in OSA patients (7-60\%; Harris et al., 2009; Saunamaki \& Jehkonen, 2007). Correlational studies of OSA severity and scores on depression scales have had mixed results, with some studies showing a more consistent relationship in women (Harris et al., 2009). One longitudinal study found increased risk of developing depression with increasing OSA severity (Peppard et al., 2006).

In the present study, we found that OSA is a risk factor for MDD (adjusted IRR = 3.58). Due to the high prevalence of OSA and SDB, we were able to separately evaluate the risk of OSA and other breathing disorders. We found that OSA is a stronger risk factor for depression than other types of SDB (adjusted IRR 3.58 compared to 1.98 ). Our results suggest that breathing difficulties caused by obstruction of the airways confer greater risk to developing depression.

\section{Inorganic Versus Organic Sleep Disorders}

The risk for depression was significantly higher for inorganic disorders (adjusted IRR $=4.09,95 \%$ confidence interval $(\mathrm{CI})[3.14$, 5.33]) than for organic disorders (adjusted $I R R=2.20,95 \% C I$ $[2.01,2.41])$. This is perhaps not surprising given that inorganic disorders are those where there is a mental rather than physical origin. This may reflect real differences in risk because of shared etiology, but it could also reflect differences in treatment and care, whereby patients diagnosed with an inorganic disorder are more likely to be referred for a psychiatric evaluation.

\section{Interval Between Sleep Disorders and Development of Depression}

Further analyses showed that the risk of developing depression is highest in those recently diagnosed with a sleep disorder. But those who do not develop depression in the year subsequent to developing a sleep disorder remain at risk. It is therefore important to monitor patients with sleep disorders even years after they were diagnosed in order to try to minimize the risk of developing depression. The increased risk that remains may be specific to those whose sleep disorder is refractory to treatment or who suffer a relapse. It was not possible to test such a hypothesis in the present study. The results of these analyses should be interpreted with caution because they represent the time that a person presented to a hospital and received a diagnosis. The symptoms may have started 
some time prior to the time of diagnosis and may have required a person to reach a threshold of symptom severity before seeking help from a general or psychiatric hospital. In addition, patients diagnosed with a sleep disorder may have been monitored for a period of time by doctors for depression, thus increasing the likelihood that they would receive a diagnosis soon after diagnosis with a sleep disorder.

\section{Prevalence of Sleep Disorders in Danish Registers}

While there are myriad advantages of using register data, one limitation of the study is that the prevalences of sleep disorders in the registers are much lower than those found in other studies. This limitation means that it was not possible to investigate the risk associated with each specific sleep disorder diagnosis. Data from the health registers come only from general and psychiatric hospitals. General practitioners (GPs) represent the first line of care in Denmark, and it is by referral from a GP that a patient goes to the hospital. For individuals suffering with a sleep disorder, it is likely that they will first seek advice from a GP, who may suggest a course of treatment such as pharmaceutical intervention in the case of insomnia or narcolepsy.

Likewise, for those suffering from depression, a GP may prescribe antidepressants or another therapy that helps to manage the condition. Only in instances where there is significant worsening in symptoms might someone be referred to a hospital for treatment as an inpatient or outpatient. Information on diagnoses given by GPs is not available in the registers and is a significant limitation of this study. A previous evaluation of the prevalence of depression in sleep disorder patients found that while the overall prevalence of depression as measured by a score of $>10$ on the Beck Depression Scale was high (e.g., $60.5 \%$ for insomnia and $41 \%$ for OSA), the rate of moderate-severe depression was much lower (1.4\% for insomnia and 1.6\% for OSA; Vandeputte \& de Weerd, 2003).

There may be other reasons as to why the prevalence rates of sleep disorders are low compared to estimates in other populations. One possible explanation is that there is not enough recognition of sleep disorders as distinct disorders in Denmark, and that in most cases, sleep complaints are considered secondary to a primary diagnosis of a psychiatric disorder. The sleep disorder cases found here may present without any psychiatric symptoms.

\section{Conclusions}

Using data from Danish population registers, we show that all categories of sleep disorder are risk factors for incident MDD. Insomnia and circadian rhythm disorders have the highest associated risk, while narcolepsy and SDB disorders excluding OSA have the lowest associated risk. We found differences in risk between men and women for insomnia, OSA and sleep movement disorders. Furthermore, we found that there is a moderate decrease in risk of depression as the time since diagnosis of a sleep disorder decreases, but that most cases of depression were diagnosed more than a year after diagnosis of a sleep problem. Our results have implications for clinical practice. Depression screening should be considered for patients with sleep disorders, and where possible, long-term follow-up for mental health problems is advisable. Differences in risks between genders should also be considered in clinical practice.

Supplementary material. To view supplementary material for this article, please visit https://doi.org/10.1017/thg.2019.22.
Acknowledgments. The study was supported by the National Medical Research Council of Australia (NHMRC) (Grant: 1087889, Fellowships: 1053639 and 1078901), the Lundbeck Foundation Initiative for Integrative Psychiatric Research(iPSYCH), the Stanley Medical Research Institute (NCRR) and Aarhus University (CIRRAU). The funding bodies had no role in the design of the study

Conflict of interest. None.

\section{References}

American Psychiatric Association. (2013). Diagnostic and statistical manual of mental disorders (5th ed.). Washington, DC: Author.

Baglioni, C., Battagliese, G., Feige, B., Spiegelhalder, K., Nissen, C., Voderholzer, U., ... Riemann, D. (2011). Insomnia as a predictor of depression: A meta-analytic evaluation of longitudinal epidemiological studies. Journal of Affective Disorders, 135, 10-19.

Beauchemin, K. M., \& Hays, P. (1996). Dreaming away depression: The role of REM sleep and dreaming in affective disorders. Journal of Affective Disorders, $41,125-133$.

Becker, P. M., \& Sharon, D. (2014). Mood disorders in restless legs syndrome (Willis-Ekbom disease). Journal of Clinical Psychiatry, 75, e679-e694.

Borgan, O., Goldstein, L., \& Langholz, B. (1995). Methods for the analysis of sampled cohort data in the Cox proportional hazards model. Annals of Statistics, 23, 1749-1778.

Breslau, N., Roth, T., Rosenthal, L., \& Andreski, P. (1996). Sleep disturbance and psychiatric disorders: A longitudinal epidemiological study of young adults. Biological Psychiatry, 39, 411-418.

Christensen, H., Batterham, P. J., Gosling, J. A., Ritterband, L. M., Griffiths, K. M., Thorndike, F. P., . . Mackinnon, A. J. (2016). Effectiveness of an online insomnia program (SHUTi) for prevention of depressive episodes (the GoodNight Study): A randomised controlled trial. Lancet Psychiatry, 3, 333-341.

Ford, D. E., \& Kamerow, D. B. (1989). Epidemiologic study of sleep disturbances and psychiatric disorders. An opportunity for prevention? JAMA, 262, 1479-1484.

Fortuyn, H. A., Lappenschaar, M. A., Furer, J. W., Hodiamont, P. P., Rijnders, C. A., Renier, W. O., ... Overeem, S. (2010). Anxiety and mood disorders in narcolepsy: A case-control study. General Hospital Psychiatry, $32,49-56$.

Germain, A., \& Kupfer, D. J. (2008). Circadian rhythm disturbances in depression. Human Psychopharmacology, 23, 571-585.

Harris, M., Glozier, N., Ratnavadivel, R., \& Grunstein, R. R. (2009). Obstructive sleep apnea and depression. Sleep Medicine Reviews, 13, 437-444.

Kales, J. D., Kales, A., Soldatos, C. R., Caldwell, A. B., Charney, D. S., \& Martin, E. D. (1980). Night terrors. Clinical characteristics and personality patterns. Archives of General Psychiatry, 37, 1413-1417.

Krishnan, R. R., Volow, M. R., Miller, P. P., \& Carwile, S. T. (1984). Narcolepsy: Preliminary retrospective study of psychiatric and psychosocial aspects. American Journal of Psychiatry, 141, 428-431.

Laursen, T. M., Musliner, K. L., Benros, M. E., Vestergaard, M., \& MunkOlsen, T. (2016). Mortality and life expectancy in persons with severe unipolar depression. Journal of Affective Disorders, 193, 203-207.

Llorente, M. D., Currier, M. B., Norman, S. E., \& Mellman, T. A. (1992). Night terrors in adults: phenomenology and relationship to psychopathology. Journal of Clinical Psychiatry, 53, 392-394.

Lynge, E., Sandegaard, J. L., \& Rebolj, M. (2011). The Danish National Patient Register. Scandinavian Journal of Public Health, 39, 30-33.

Mors, O., Perto, G. P., \& Mortensen, P. B. (2011). The Danish Psychiatric Central Research Register. Scandinavian Journal of Public Health, 39, 54-57.

Munk-Jorgensen, P., \& Mortensen, P. B. (1997). The Danish Psychiatric Central Register. Danish Medical Bulletin, 44, 82-84.

Ohayon, M. M. (2013). Narcolepsy is complicated by high medical and psychiatric comorbidities: A comparison with the general population. Sleep Medicine, 14, 488-492.

Pedersen, C. B. (2011). The Danish Civil Registration System. Scandinavian Journal of Public Health, 39, 22-25. 
Pedersen, C. B., Gotzsche, H., Moller, J. O., \& Mortensen, P. B. (2006). The Danish Civil Registration System. A cohort of eight million persons. Danish Medical Bulletin, 53, 441-449.

Pedersen, C. B., Mors, O., Bertelsen, A., Waltoft, B. L., Agerbo, E., McGrath, J. J., ... Eaton, W. W. (2014). A comprehensive nationwide study of the incidence rate and lifetime risk for treated mental disorders. JAMA Psychiatry, 71, 573-581.

Peppard, P. E., Szklo-Coxe, M., Hla, K. M., \& Young, T. (2006). Longitudinal association of sleep-related breathing disorder and depression. Archives of Internal Medicine, 166, 1709-1715.

Perlis, M. L., Smith, L. J., Lyness, J. M., Matteson, S. R., Pigeon, W. R., Jungquist, C. R., \& Tu, X. (2006). Insomnia as a risk factor for onset of depression in the elderly. Behavioral Sleep Medicine, 4, 104-113.
Petersson, F., Baadsgaard, M., \& Thygesen, L. C. (2011). Danish registers on personal labour market affiliation. Scandinavian Journal of Public Health, 39, 95-98.

Roy, A. (1976). Psychiatric aspects of narcolepsy. British Journal of Psychiatry, 128, 562-565.

Saunamaki, T., \& Jehkonen, M. (2007). Depression and anxiety in obstructive sleep apnea syndrome: A review. Acta Neurologica Scandinavica, 116, 277-288.

Vandeputte, M., \& de Weerd, A. (2003). Sleep disorders and depressive feelings: A global survey with the Beck depression scale. Sleep Medicine, 4, 343-345.

Vourdas, A., Shneerson, J. M., Gregory, C. A., Smith, I. E., King, M. A., Morrish, E., \& McKenna, P. J. (2002). Narcolepsy and psychopathology: Is there an association? Sleep Medicine, 3, 353-360. 